\title{
Finding erythroid stress progenitors: cell surface markers revealed
}

\section{Peng Ji}

Department of Pathology, Feinberg School of Medicine, Northwestern University, Chicago, IL and Robert H. Lurie Comprehensive Cancer Center, Northwestern University, Chicago, IL, USA

E-mail: PENG JI - peng-ji@fsm.northwestern.edu

doi:10.3324/haematol.2020.262493

T he constant production of red blood cells maintains the hematologic homeostasis during steady state in mammals. ${ }^{1}$ Under stress conditions, such as bleeding or hemolysis, the increased perfusion pressure enables the switch of steady erythropoiesis to stress erythropoiesis. Research over the past few decades led to our increased understanding of the molecular mechanisms in stress erythropoiesis, especially in murine models. ${ }^{2}$ However, finding the stress erythroid progenitor cells for detailed mechanistic investigation remains a demanding task. In this issue of Haematologica, Singbrant et al. ${ }^{3}$ discovered cell surface markers that could aid the identification of these progenitors.

Bone marrow is the major organ that produces red blood cells in adult mammals under steady state. During stress erythropoiesis in mouse models, red cell production switches to the spleen and liver. Accumulating evidence demonstrated that bone morphogenetic protein 4 (BMP4) signaling, along with erythropoietin (Epo), Hedgehog, stem cell factor (SCF), and hypoxia, play critical roles during stress erythropoiesis..$^{4-8}$ These signaling pathways ensure a rapid response of the hematopoietic system to produce a large quantity of erythrocytes for tissue hypoxia. The erythroid progenitors responsive for stress erythropoiesis are burst-forming unit-erythroid progenitors (BFU-E) found in murine spleens. Previous reports have shown that these splenic stress BFU-E express immature cell surface marker c-Kit and low levels of erythroid markers CD71 and Ter119. . Further efforts were applied to expand the stress BFU-E population in vitro using additional markers such as CD34 and CD133. ${ }^{10}$ However, this progenitor population is rather heterogenous with a low percentage of cells being stress BFU-E.

To identify and enrich the stress erythroid progenitors, Singbrant et $a .^{3}$ used an irradiation-induced anemia mouse model to mimic stress erythropoiesis. The authors previously showed that fetal erythroid BFU-E can be identified with high purity as lineage cKit ${ }^{+}$and CD71/CD24a ${ }^{\text {low }}$ Sca1 CD34 in mouse fetal liver. ${ }^{11}$ Since adult stress erythropoiesis in murine models closely resembles fetal erythropoiesis, the authors determined whether splenic stress erythroid progenitors could also be enriched using these and additional markers. This approach led to the discovery that stress BFU-E could be further enriched from Lineage $\mathrm{cKit}^{+} \mathrm{CD} 71 / \mathrm{CD} 24 \mathrm{a}^{\text {low }}$ cells

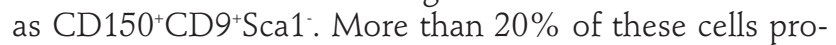
duced large BFU-E colonies, which represented over 100fold improvement of purity compared to previous methods. In addition to the identification of high purity stress BFU-E, multi-potent stress progenitors (stress-MPP) that give rise to stress BFU-E, and stress colony-forming uniterythroid progenitors (stress-CFU-E) were also identified

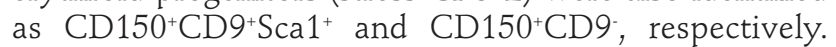

Using an elegant in vivo tracing technology with Kusabira orange (KuO) mice, the authors further demonstrated that stress-BFU-E and stress-CFU-E harbor a short-term radio-protective capacity by providing a transient wave of reconstitution in the peripheral blood and spleen. Stress-MPP follow the short-term wave and provide the multi-lineage reconstitution in the peripheral blood, spleen, and bone marrow.

Gene expressing profiling analyses further demonstrated that stress MPP and BFU-E in murine spleen express target genes of $\mathrm{BMP}$, which is consistent with its role in stress erythropoiesis. The authors extended these findings by showing that mice transplanted with BMP receptor II deficient donor bone marrow cells had smaller spleens and a significant reduction in spleen cells. BMP receptor II deficient bone marrow cells also showed significantly decreased potential to form stress BFU-E from lineage $\mathrm{cKit}^{+}$progenitors. These studies confirmed the critical role of BMP signaling in stress erythropoiesis in murine models from a genetic approach.

Interestingly, the authors also found $\mathrm{CD} 150^{+} \mathrm{CD} 9+\mathrm{BFU}-$ E progenitors in steady-state bone marrow but not the spleen, demonstrating that these markers are useful to identify BFU-E progenitors in both steady-state and stress conditions. Previous studies indicated that steady BFU-E migrate from the bone marrow to the spleen during stress erythropoiesis to become stress BFU-E, ${ }^{12}$ while more recent findings suggest that endogenous splenic stress BFU-E during stress erythropoiesis are distinctive from steady BFU-E. The $\mathrm{CD} 150^{+} \mathrm{CD}^{+}{ }^{+} \mathrm{BFU}-\mathrm{E}$ progenitors Singbrant et $a l .^{3}$ discovered in both steady state and stress conditions would be helpful to resolve this contradiction in future studies.

Using RNA sequencing and gene set enrichment analyses, the authors further revealed that steady- and stressBFU-E exhibited a large overlap in the transcriptome. The differences are mainly in genes associated with BMP and glucocorticoid signaling, proliferation, maturation block, and erythropoiesis that are highly expressed in stress BFU-E in the spleen. The most downregulated genes in stress BFU-E include those associated with myeloid cell development and immune response. While the transcriptional landscapes of steady- and stress-BFU-E are similar, another interesting finding in this study is that there is differential chromatin accessibility in the distal elements in stress erythroid progenitors. Transcriptional regulators CTCF and ERG are significantly enriched in open chromatin regions in stress-BFU-E, indicating their potential roles in regulating stress erythropoiesis.

The findings by Singbrant et al. ${ }^{3}$ provide the field with an important tool to isolate stress-BFU-E in mice. This allows researchers to investigate stress erythropoiesis in a variety of mouse model systems that mimic disease con- 


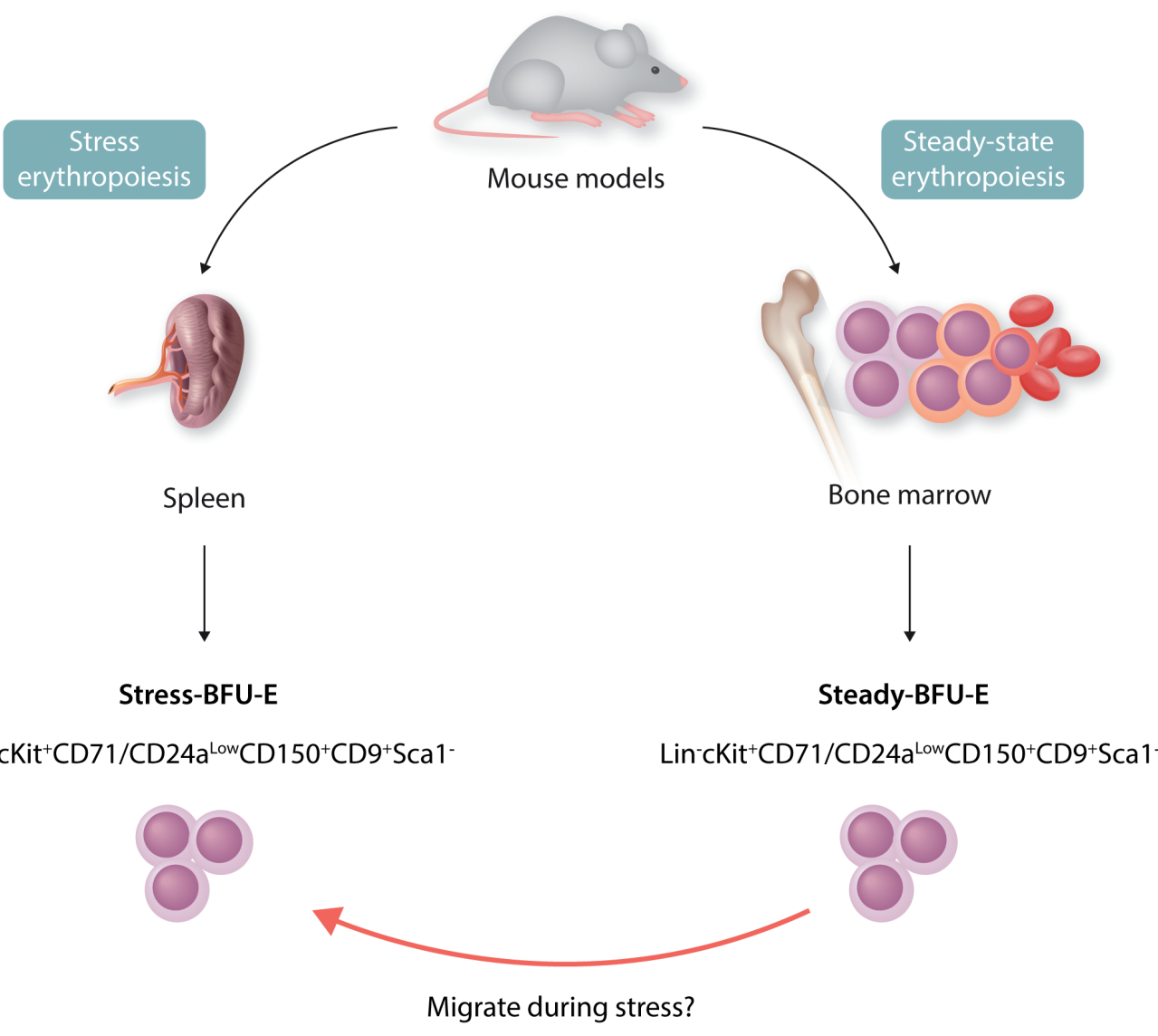

Figure 1. Schematic view of steady-state and stress erythropoiesis in mouse models. Mouse steady-state and stress erythropoiesis occur primarily in the bone marrow and spleen, respectively. Singbrant et al. ${ }^{3}$ discovered cell surface markers that are common in steady- and stress-burst-forming unit-erythroid progenitors (BFU-E). It remains to be investigated whether and how steady-BFU-E migrate from the bone marrow to the spleen during stress.

ditions with acute anemia. With the relatively more specific markers to enrich steady- and stress-BFU-E, a detailed mechanistic investigation of erythroid lineage commitment becomes more feasible. In this aspect, Singbrant et $a .^{3}$ found that in addition to genes downstream of BMP signaling, genes that are regulated by Cbfa2t3 (also known as Eto2) were also upregulated in stress erythroid progenitors. Cbfa2t 3 is a transcriptional co-repressor that maintains the primed state of erythroid progenitors and is known to be involved in stress erythropoiesis. ${ }^{13,14}$ Future studies on how $\mathrm{Cbfa} 2 \mathrm{t} 3$ regulates stress erythropoiesis and whether the $\mathrm{Cbfa} 2 \mathrm{t} 3$ transcriptional corepressor complex cross talks with BMP signaling would be interesting to pursue.

One of the key remaining questions is whether these or similar markers can be used to identify human stress erythroid progenitors. The field of stress erythropoiesis relies heavily on mouse models. Several important differences are present between mouse and human. In mouse, the nature of the hypercellular bone marrow provides limited spaces for the expansion of an erythroid lineage during stress, which forces the spleen to become a major extramedullary erythropoiesis organ. However, this phenomenon is not common in humans. ${ }^{15}$ Although studies have shown that BMP signaling is also involved in human stress erythropoiesis in vitro, ${ }^{10}$ it is unclear whether the same is true in vivo. Exploration of markers and signaling pathways in stress erythroid progenitors in human, or other model systems such as rats, would be the necessary next step.

\section{References}

1. Hattangadi SM, Wong P, Zhang L, Flygare J, Lodish HF. From stem cell to red cell: regulation of erythropoiesis at multiple levels by multiple proteins, RNAs, and chromatin modifications. Blood. 2011:118(24):6258-6268

2. Bennett LF, Liao C, Paulson RF. Stress erythropoiesis model systems. Methods Mol Biol. 2018;1698:91-102.

3. Singbrant S, Mattebo A, Sigvardsson M, Strid T, Flygare J. Prospective isolation of radiation induced erythroid stress progenitors reveals unique transcriptomic and epigenetic signatures enabling increased erythroid output. Haematologica. 2020; 105(11):2561-2571.

4. Perry JM, Harandi OF, Paulson RF. BMP4, SCF, and hypoxia cooperatively regulate the expansion of murine stress erythroid progenitors. Blood. 2007:109(10):4494-4502.

5. Lenox LE, Perry JM, Paulson RF. BMP4 and Madh5 regulate the erythroid response to acute anemia. Blood. 2005;105(7):2741-2748.

6. Porayette P, Paulson RF. BMP4/Smad5 dependent stress erythropoiesis is required for the expansion of erythroid progenitors during fetal development. Dev Biol. 2008;317(1):24-35.

7. Paulson RF, Shi L, Wu DC. Stress erythropoiesis: new signals and new stress progenitor cells. Curr Opin Hematol. 2011;18(3):139-145.

8. Perry JM, Harandi OF, Porayette P, Hegde S, Kannan AK, Paulson RF. Maintenance of the BMP4-dependent stress erythropoiesis pathway in the murine spleen requires hedgehog signaling. Blood. 2009;113(4):911-918.

9. Harandi OF, Hedge S, Wu DC, McKeone D, Paulson RF. Murine ery- 
throid short-term radioprotection requires a BMP4-dependent, selfrenewing population of stress erythroid progenitors. J Clin Invest. 2010;120(12):4507-4519.

10. Xiang J, Wu DC, Chen Y, Paulson RF. In vitro culture of stress erythroid progenitors identifies distinct progenitor populations and analogous human progenitors. Blood. 2015;125(11):1803-1812.

11. Flygare J, Rayon Estrada V, Shin C, Gupta S, Lodish HF. HIF1alpha synergizes with glucocorticoids to promote BFU-E progenitor selfrenewal. Blood. 2011;117(12):3435-3444

12. Hara H, Ogawa M. Erthropoietic precursors in mice with phenylhy- drazine-induced anemia. Am J Hematol. 1976;1(4):453-458.

13. Chyla BJ, Moreno-Miralles I, Steapleton MA, et al. Deletion of Mtg16, a target of $\mathrm{t}(16 ; 21)$, alters hematopoietic progenitor cell proliferation and lineage allocation. Mol Cell Biol. 2008;28(20):62346247.

14. Stadhouders R, Cico A, Stephen T, et al. Control of developmentally primed erythroid genes by combinatorial co-repressor actions. Nat Commun. 2015;6:8893.

15. Zhang J, Liu Y, Han X, et al. Rats provide a superior model of human stress erythropoiesis. Exp Hematol. 2019;78:21-34 e23.

\section{COVID-19 and sickle cell disease}

\section{Laurel A. Menapace and Swee Lay Thein}

Sickle Cell Branch, National Heart Lung and Blood Institute, National Institutes of Health, Bethesda, MD, USA

E-mail: SWEE LAYTHEIN - sl.thein@nih.gov

doi:10.3324/haematol.2020.255398

T he spread of the novel SARS-CoV-2 coronavirus (COVID-19) has resulted in widespread lockdowns and an unprecedented number of deaths globally.,2 The pandemic has posed unique challenges to healthcare providers involved in the care of individuals with chronic conditions, balancing maintenance of necessary care with appropriate precautions to reduce their exposure to infection. Patients with sickle cell disease (SCD) present multiple challenges due to the complexity of their condition, disease-related comorbidities, and need for frequent medical interventions. To date, there has been a paucity of published data on how COVID-19 may impact morbidity and mortality in SCD patients.

In this edition of Haematologica, Charkravorthy and colleagues report associated outcomes in 10 UK patients infected with SARS-CoV-2. ${ }^{3}$ All patients had hemoglobin $(\mathrm{Hb})$ SS disease with pre-existing co-morbidities, despite of this nine patients made a full recovery without receiving COVID-19 directed therapies. Five patients were hospitalized and treated supportively; two patients who had a cough and hypoxia received early preventive simple transfusions; five patients were managed at home via close telephone contact. Of the 10 patients, seven were female (median age of 37 years); two received hydroxyurea therapy and seven received regular blood transfusions (four exchange and three simple transfusions). Given that COVID-19 is an acute infectious pneumonia, most experts anticipated that SARS-CoV-2 infection would trigger acute chest syndrome (ACS) yet only one patient who died in this series had significant respiratory complications. The patient who died was a 54-year-old female with severe asthma, alloimmunization, and a history of delayed hemolytic transfusion reactions which prevented routine transfusion. The patient had lymphopenia, thrombocytopenia and elevated C-reactive protein (CRP), which have been identified as poor prognostic markers in COVID-19 patients. ${ }^{4}$ The authors acknowledge that the demographic and treatment characteristics of this cohort may have influenced the observed outcomes.

This edition also includes a report of 195 cases of suspected or confirmed SARS-CoV-2 infection as captured by 10 regional centers in a real time survey over a 4 -week period in the UK. ${ }^{5}$ Patients with SCD represented the majority of cases ( $\mathrm{n}=166,85.1 \%$ ) with $\mathrm{Hb}$ SS disease as the most common genotype (Hb SS $64.1 \%$; Hb SC $15.4 \%$; other genotypes $5.6 \%$ ) followed by thalassemia $(n=26$, $13.3 \%)$ and rare inherited anemias $(n=3,1.5 \%)$. While the incidence of ACS in the SCD cohort was not specified, red cell exchange was performed in 46 patients during the course of infection. A total of eight SCD patients required mechanical ventilatory support in the setting of respiratory failure; 11 of 13 deaths attributed to COVID-19 occurred in patients with SCD. A subset analysis of 76 hospitalized SCD patients with PCR confirmed SARSCoV-2 showed that the patient age was significantly associated with mortality, with the most deaths occurring in those aged 50 and older $(n=4)$. The authors noted that three patients aged 20-39 years in the subset succumbed to COVID-19 although details regarding the comorbid medical conditions were not provided. Unexpectedly, mortality was higher in females and was not associated with severity of disease but observed differences were insignificant. The cases reported to date represent $1.2 \%$ of the estimated 13,655 individuals with SCD per registry data in England. There was a low incidence of infection in children $(n=20)$ and there were no pediatric deaths. Definitive conclusions regarding COVID-19 mortality rates in SCD cannot be drawn given inherent ascertainment bias and missing data in the survey.

The mild clinical course of COVID-19 in the King's College Hospital SCD patients ${ }^{3}$ was similarly encountered in a case series of four patients from Chicago, ${ }^{6}$ all of whom presented with acute pain. Of the four patients, three received the usual supportive care but one patient, a 32-year-old male with Hb SS, developed ACS requiring intubation and exchange transfusion.

Two other centers reported COVID-19 pneumonia causing ACS in SCD patients. A 21-year-old male with $\mathrm{Hb} S / \beta^{\circ}$ thalassemia on hydroxyurea therapy presented with acute chronic left hip pain and subsequently developed a cough with progressive hypoxia and evidence of new pulmonary infiltrates during his hospital course.? SARS-CoV-2 PCR testing was positive during the second week of admission. In addition to antibiotic therapy, the patient was initiated 